\title{
Interactive comment on "A land surface model combined with a crop growth model for paddy rice (MATCRO-Rice Ver. 1) - Part I: Model description" by Yuji Masutomi et al.
}

\section{Anonymous Referee \#2}

Received and published: 1 May 2016

This paper is the description of the model of a land surface model (MATCRO) combined with a crop growth model for paddy rice. The paper well describes the underlying scientific basis. Combining land surface model with crop growth model is an important task to understand the interaction between climate and cropland. The paper also well explains the frameworks of the model. The model description part of this paper is very understandable. The model is designed for consistently simulating latent and sensible heat fluxes, net carbon flux, and crop yield by exchanging variables between the land surface model and crop growth model. The main frame work of the land surface module of this model is the same with original MATCRO, except for the addition of surface water above the soil. The crop growth model module is the combination of several models,

Printer-friendly version

Discussion paper 
for example, sun-shade model for the assimilation module, a simple canopy model by Watanabe and Ohtani (1995) for calculating PAR, and MACROS Penning de Vries et al., 1989 and ORYZA2000 (Bouman et al., 2001) for crop growth.

I feel that the choices of these modules are appropriate for the purpose of the model. However, the calibrating method of parameters is explained in another submitted paper (Masutomi et al. 2016 in review). The examples of the output of this model are not provided in the current paper and these are provided in the accompanied paper.

It is my understanding that examples of model output should be provided, with evaluation against standard benchmarks, observations in GMD. There appears to be no reason to divide the model description paper to two papers because both the model description part (this paper) and the validation part (another submitted paper) are not so long. However, if dividing the study into two papers is acceptable, I think this paper is acceptable. The model description part is well explained. The equations described in this paper have no mistake.

\section{Specific Comments}

p. 5, I. 11: Is this assumption appropriate? The model that the authors are developing is rice specific model. However, the leaf orientation of most Poaceae species would not be random. The required preciseness for the leaf orientation may depend on the purpose of the model (or temporal resolution), but the precise description of the leaf orientation may be needed if the purpose of the model is the estimation of hourly fluctuation of the fluxes. If the purpose of the model is the estimation of crop yield for example, the assumption of the leaf orientation may not have critical effect on the estimation. The authors should add the discussion of the appropriateness of the assumption.

p. 6, I. 19-20: Please explain in detail.

p. 11, I. 28: The down-regulation effect of photosynthesis has a very profound effect

Printer-friendly version

Discussion paper
Interactive comment

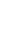


on crop growth. The parameters relevant to photosynthesis down-regulation in Arora et al. (2009) are calculated using mainly plants other than rice. Therefore, the authors should explain the applicability of the parameter values to rice.

Eq. 69-71: Please change the variable name of "Qt". The The character "Q" is already used for the photon flux density.

All equations: Italic should be used for only scalars in principle. For example, it may be preferable not to use Italic for the subscript "c" of "Hc" if "c" is not scalar value. Moreover, upright font (not italic) should be used for multi-letter variables (for example, "Rnc"). Please recheck almost of all subscripts and superscripts of the equations.

Interactive comment on Geosci. Model Dev. Discuss., doi:10.5194/gmd-2016-28, 2016. 